\title{
Simultaneous Tunable Enhancement of SHG and THG in Graphene Optical Gratings
}

\author{
Jian Wei You and Nicolae C. Panoiu \\ Department of Electronic and Electrical Engineering, University College London, London, UK, WC1E 7JE \\ e-mail address: j.you@ucl.ac.uk and n.panoiu@ucl.ac.uk
}

\begin{abstract}
Employing gate voltage tunable and geometry dependent plasmon resonances of graphene ribbons, we engineer dual graphene optical gratings that can be used to simultaneously achieve orders-of-magnitude enhancement of both the SHG and THG intensities.

OCIS codes: $190.0190,160.3918,240.6680$
\end{abstract}

\section{Introduction}

Because of large optical field enhancements achievable in graphene via excitation of surface plasmons, this truly twodimensional (2D) physical system [1] provides a promising material platform for enhancing nonlinear optical interactions at the nanoscale [2-4]. In this paper, we demonstrate that by matching plasmon resonances in mutually interacting graphene gratings separated by a thin dielectric spacer, the second-harmonic $(\mathrm{SH})$ and third-harmonic $(\mathrm{TH})$ can be simultaneously enhanced by more than eight orders of magnitude as compared to that in a graphene sheet. Choosing which nonlinear optical effect is enhanced can be conveniently done by gate voltage tuning. To be more specific, employing the dependence of the resonance wavelength of localized plasmons of graphene ribbons on their width, we design a graphene bilayer grating in such a way that the resonance wavelength of the first-order localized plasmons in one grating is equal to half (for $\mathrm{SH}$ ) and a third (for $\mathrm{TH}$ ) of that of the first-order localized plasmons in an adjacent grating that is optically coupled to the first one. This finding is particularly relevant in the context of technological applications, as graphene plays an increasingly important role in optoelectronics, chiefly due to the remarkable tunability of its optical properties via chemical doping or electric gating. In addition, graphene structures support localized and propagating surface plasmons, whose excitation is accompanied by a significant enhancement of the optical near-field. This unique potential to control at deep-subwavelength the optical field has open up a broad array of technological applications, including optical modulation, sensing, and ultrafast active photonic devices $[5,6]$.

\section{Linear Optical Response of Single and Dual Graphene Gratings}

The physical structure considered in this study consists of two-layer graphene gratings, also called dual gratings, depicted in the inset of Fig. 1(a), where two single graphene gratings are placed at the top and bottom facets of a spacer made of glass with relative permittivity, $\epsilon_{s}=2.25$. In our design, the two periods are $\Lambda_{1}=100 \mathrm{~nm}$ and $\Lambda_{1}=50 \mathrm{~nm}$, the chemical potential of graphene is $0.6 \mathrm{eV}$, and the carriers relaxation time is $0.25 \mathrm{ps}$. The widths of the graphene ribbons, $W_{1}$ and $W_{2}$, and the thickness, $H$, of the spacer are quantities we optimize to achieve a so-called double resonance [4], that is to have a plasmon mode at the fundamental frequency (FF) and also a plasmon mode at the SH or TH. To model the linear and nonlinear optical response of graphene gratings, we used a numerical method implementing a combination of the generalized-source algorithm and finite-different time-domain method [3].
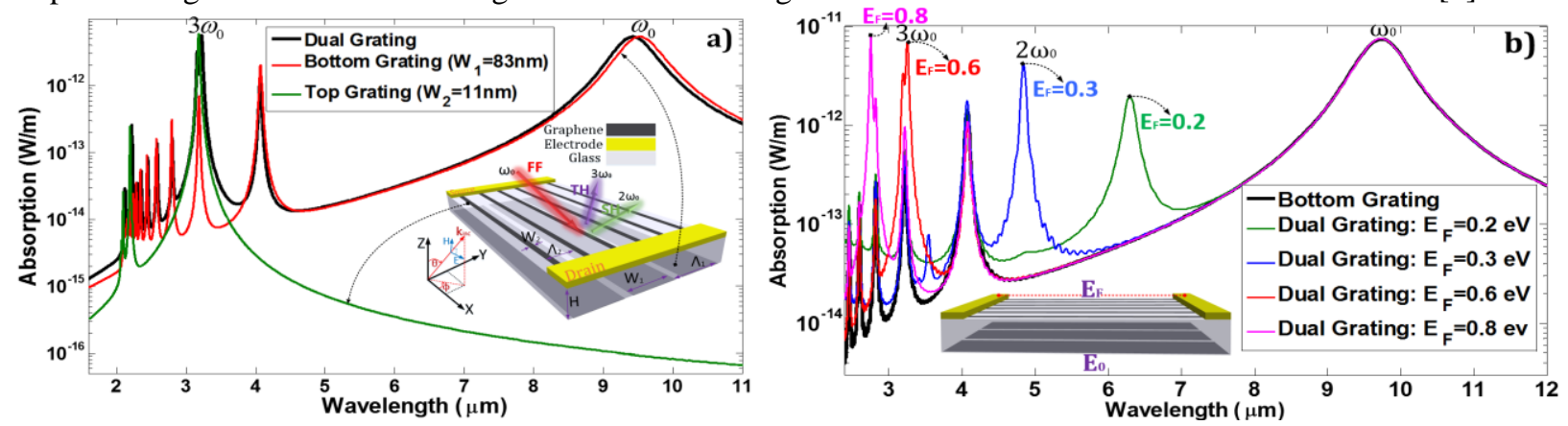

Fig. 1. Geometrical configuration and optimization of a two-layer graphene grating: (a) Enhancement of THG in a two-layer graphene grating via engineering fundamental plasmon resonances at $\omega_{0}$ and $3 \omega_{0}$. (b) Illustration of the tunability of the nonlinear optical response of the proposed two-layer graphene optical grating and mechanism to achieve simultaneous enhancement of both SHG and THG in the same graphene grating.

We begin our analysis by first considering only the bottom graphene grating and engineering the width $\left(W_{1}\right)$ to achieve a double resonance at TH. As illustrated by the red curve in Fig. 1(a), the bottom graphene ribbon supports a series of plasmon resonances, seen in this figure as strong peaks. Moreover, there is a strong peak located exactly at a 
third of the resonance wavelength of the first-order plasmon resonance, when $W_{1}=83 \mathrm{~nm}$. This proves that there is a double plasmon resonance at this specific width for a single graphene grating. To further enhance the THG at this double resonance, we place another graphene grating on top of the glass spacer, and calculate the linear absorption spectra at different width $\left(W_{2}\right)$. The spectrum shown with green line in Fig. 1(a) suggests that the first-order plasmon resonance of the top graphene grating with $W_{2}=10.9 \mathrm{~nm}$ coincides with a third of the resonance wavelength of the first-order plasmon of the bottom graphene grating. Thus, to summarize our approach, the bottom graphene grating has an intrinsic double plasmon resonance, meaning the wavelength of the third-order plasmon is a third of that of the fundamental plasmon, whereas additional THG enhancement is provided by the top grating, for which the wavelength of the fundamental plasmon is equal to a third of that of the fundamental plasmon of the bottom grating. Note that the same procedure can be used to design a dual graphene grating for enhancement of SHG, too.

Importantly, the graphene grating optimized for enhancement of THG can be used to enhance the SHG, too, by simply tuning the gate voltage applied to the top grating, as per Fig. 1(b). Thus, by decreasing the chemical potential of the top grating from $0.6 \mathrm{eV}$ to $0.3 \mathrm{eV}$, the resonance wavelength of the first-order plasmon of this grating is increased from $\lambda_{0} / 3$ to $\lambda_{0} / 2$, where $\lambda_{0}$ is the resonance wavelength of the first-order plasmon of the bottom graphene grating.

\section{Optical Nonlinearity Enhancement: Results and Discussion}

The design ideas for simultaneous enhancement of SHG and THG are validated by the nonlinear spectra plotted in Fig. 2. Thus, the single grating optimized for enhancement of THG, namely a grating possessing an intrinsic double resonance as reported in [4], has period $\Lambda_{1}=100 \mathrm{~nm}$ and width $W_{1}=85 \mathrm{~nm}$. It can be seen from Fig. 2(a) that, as compared to a uniform graphene sheet, the THG in this single grating is more 6 orders of magnitude larger. By adding a top grating that has a first-order plasmon resonance at the TH, the THG in this dual graphene grating is enhanced by an additional factor of $\sim 44$, and thus by more than 8 orders of magnitude as compared to a uniform graphene sheet. Importantly, this same grating can be used to enhance the SHG, too, by tuning the chemical potential from $0.6 \mathrm{eV}$ to $0.3 \mathrm{eV}$. This is illustrated in Fig. 2(b), where we present the SHG spectra corresponding to the bottom single grating and the dual grating tuned to achieved maximum SHG intensity. More specifically, it can be clearly seen from this figure that the SHG in the dual graphene grating is enhanced by a factor of $\sim 45$, simply by tuning the gate voltage.
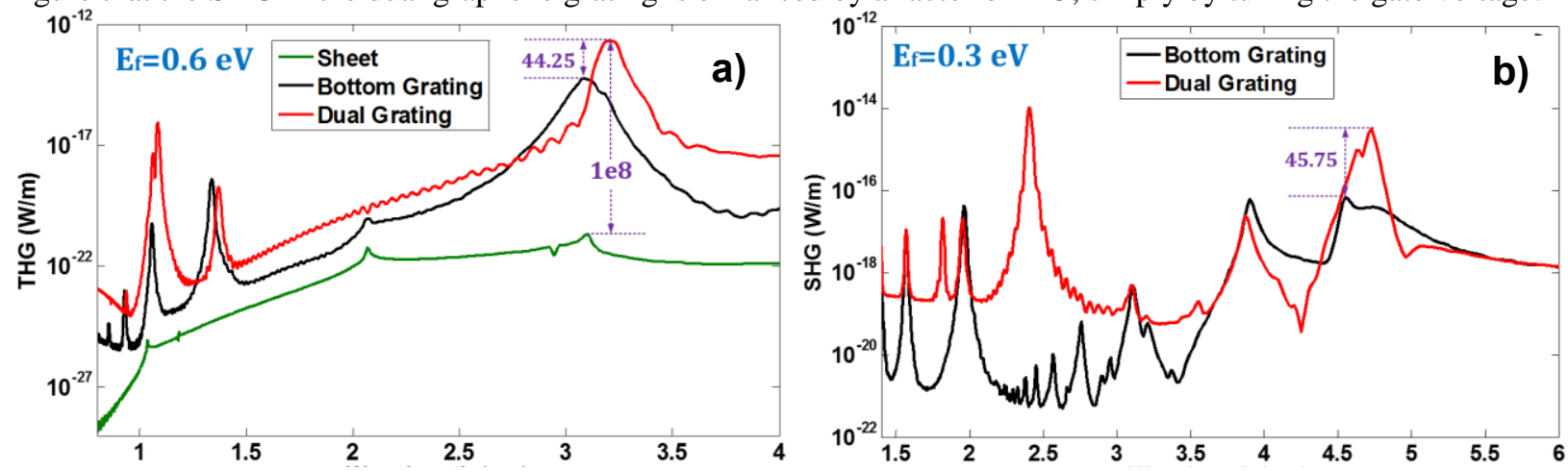

Fig. 2. Comparison between the generated nonlinear optical signal in single and optimized dual graphene gratings: (a) enhancement of second-harmonic generation and (b) enhancement of third-harmonic generation.

\section{Conclusion}

In conclusion, we proved that giant enhancement of both TGH and SHG can be achieved in the same graphene bilayer grating by properly designing the spectral location of the plasmon resonances of optically coupled graphene gratings, combined with gate voltage tuning. Because of the generality of the employed design principles, we expect that the ideas investigated here can also be applied to design other active graphene photonic nanodevices.

\section{References}

[1] K. S. Novoselov, A. K. Geim, S. V. Morozov, D. Jiang, Y. Zhang, S. V. Dubonos, I. V. Grigorieva, and A. A. Firsov. "Electric field effect in atomically thin carbon films," Science 306, 666-669 (2004).

[2] M. T. Manzoni, I. Silveiro, F. J. G. Abajo, and D. E. Chang. "Second-order quantum nonlinear optical processes in single graphene nanostructures and arrays," New J. Phys. 17, 083031 (2015).

[3] M. Weismann and N. C. Panoiu, "Theoretical and computational analysis of second- and third-harmonic generation in periodically patterned graphene and transition-metal dichalcogenide monolayers," Phys. Rev. B. 94, 035435 (2016).

[4] J. W. You, J. You, M. Weismann, and N. C. Panoiu, "Double-resonant enhancement of third-harmonic generation in graphene nanostructures," Phil. Trans. R. Soc. A. 375, 20160313 (2017).

[5] F. Bonaccorso, Z. Sun, T. Hasan, and A. C. Ferrari. "Graphene photonics and optoelectronics," Nat. Photonics 4, 611-622 (2010).

[6] F. Xia, T. Mueller, Y. M. Lin, A. Valdes-Garcia, and P. Avouris. "Ultrafast graphene photodetector," Nat. Nanotechnol. 4, 839-843 (2009).

[7] J. L. Cheng, N Vermeulen, and J. E. Sipe, “Third order optical nonlinearity of graphene,” New J. Phys. 16, 053014 (2014). 